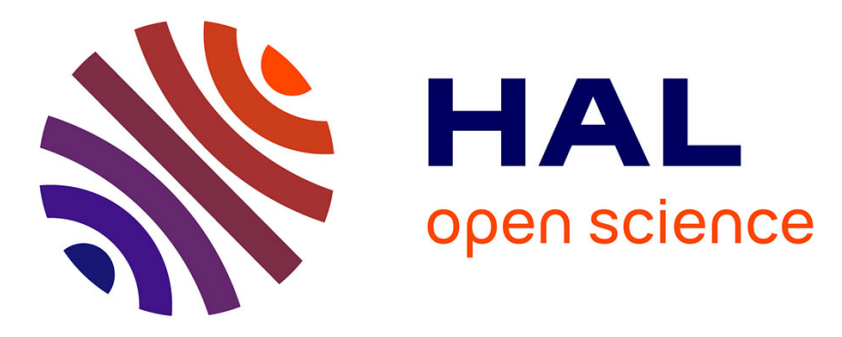

\title{
Smart Cities Connected and Autonomous Vehicles Readiness Index
}

Junaid A. Khan, Lan Wang, Eddie Jacobs, Ahmedraza Talebian, Sabyasachee Mishra, Charles A Santo, Mihalis Golias, Carmen Astorne-Figari

\section{To cite this version:}

Junaid A. Khan, Lan Wang, Eddie Jacobs, Ahmedraza Talebian, Sabyasachee Mishra, et al.. Smart Cities Connected and Autonomous Vehicles Readiness Index. ACM SCC, 2019, Portland, OR, United States. 10.1145/1122445.1122456 . hal-02291446

\section{HAL Id: hal-02291446 \\ https://hal.science/hal-02291446}

Submitted on 18 Sep 2019

HAL is a multi-disciplinary open access archive for the deposit and dissemination of scientific research documents, whether they are published or not. The documents may come from teaching and research institutions in France or abroad, or from public or private research centers.
L'archive ouverte pluridisciplinaire HAL, est destinée au dépôt et à la diffusion de documents scientifiques de niveau recherche, publiés ou non, émanant des établissements d'enseignement et de recherche français ou étrangers, des laboratoires publics ou privés. 


\title{
Smart Cities Connected and Autonomous Vehicles Readiness Index
}

\author{
Junaid Ahmed Khan, Lan Wang, Eddie Jacobs, Ahmedraza Talebian, Sabyasachee Mishra, Charles \\ A Santo, Mihalis Golias and Carmen Astorne-Figari \\ University of Memphis, Memphis, TN, USA
}

\begin{abstract}
Every major car manufacturer and leading technology company today is pursuing the development of Connected and Autonomous Vehicles (CAVs). However, given cities will be most affected by CAVs, there is an urgent need to assess whether our cities are prepared and will respond well to the advancement in CAV technology. This paper proposes the first readiness index to measure the extent to which current cities are ready for CAVs. We consider three key elements in computing a CAV readiness score: a city's policies and regulations, its physical infrastructure, and its cyber infrastructure. We identify 16 major factors related to the key elements contributing to a city's readiness, and compute the readiness index as a weighted average of these factors. Moreover, we collected survey responses regarding the importance of each factor from 13 of the most populous US cities. We also selected a metric for quantifying each factor, and collected the corresponding data from our survey and existing studies. We then leverage decision trees as a machine learning model to predict 52 major US cities' readiness for CAVs. While it is difficult to draw general conclusions on our cities' readiness due to limited data availability, our preliminary study does suggest that there is a big gap between our industry and public's interest in CAVs and the policy and infrastructure support provided by our cities. Most importantly, we believe that the proposed readiness index provides practical guidelines for policy makers and planners to improve their cities' policies and infrastructure to facilitate CAVs.
\end{abstract}

\section{KEYWORDS}

Connected and Autonomous Vehicles (CAVs), Smart Cities, Readiness Index

\section{ACM Reference Format:}

Junaid Ahmed Khan, Lan Wang, Eddie Jacobs, Ahmedraza Talebian, Sabyasachee Mishra, Charles A Santo, Mihalis Golias and Carmen AstorneFigari. 2019. Smart Cities Connected and Autonomous Vehicles Readiness Index. In Proceedings of ACM/EIGSCC Symposium On Smart Cities and Communities (SCC '19). ACM, New York, NY, USA, 8 pages. https: //doi.org/10.1145/1122445.1122456

\footnotetext{
Permission to make digital or hard copies of all or part of this work for personal or classroom use is granted without fee provided that copies are not made or distributed for profit or commercial advantage and that copies bear this notice and the full citation on the first page. Copyrights for components of this work owned by others than ACM must be honored. Abstracting with credit is permitted. To copy otherwise, or republish, to post on servers or to redistribute to lists, requires prior specific permission and/or a fee. Request permissions from permissions@acm.org.

SCC '19, Sep 10-12, 2019, Portland, OR

(C) 2019 Association for Computing Machinery.

ACM ISBN 978-1-4503-9999-9/18/06 . \$ \$15.00

https://doi.org/10.1145/1122445.1122456
}

\section{INTRODUCTION}

The increase in Connected and Autonomous Vehicles (CAVs) development by car manufacturers and technology companies can potentially have a significant impact on our cities. It will not only affect the existing urban transportation system but also impact citizens' mobility trends and lifestyle. Although the adoption of CAVs by citizens depends on their preferences, market prices, and other factors $[1,2]$, we believe that cities need to proactively plan for CAV deployment by taking the necessary steps to improve their policies and infrastructure in order to facilitate CAVs. However, there are currently no concrete methodologies to measure the extent to which our cities are ready for CAVs.

This paper presents a "CAV readiness index" to measure a city's readiness for CAV deployment. We first identified the following three key elements in facilitating CAV deployment.

(1) Policy and Regulations: a city can adopt policies and regulations that make it easier for citizens and businesses to use CAVs. For example, a city may invest in projects related to CAVs or encourage such projects by providing tax incentives. It may also establish traffic rules that privilege CAVs in certain parts of the city. Establishing and enforcing such policies and regulations typically requires a dedicated CAV department within the transportation department.

(2) Physical Infrastructure: safe operations of CAVs depend on the availability and maintenance of adequate physical infrastructure such as high quality road markings and signage, high density of Electric Vehicle (EV) charging stations, as well as other CAV compatible infrastructure.

(3) Cyber infrastructure: CAVs utilize a variety of data for decision making in their navigation including data from their own sensors, data obtained from other vehicles, and data from the Internet. Therefore, their operations depend on the Internet infrastructure, Intelligent Transportation Systems (ITS), road-side units (RSUs), data centers, and other cyber infrastructure. The availability and security of such cyber infrastructure will impact the safety of the CAVs.

We further refined the above three elements into 16 factors, and computed the readiness index as the weighted average of all factors, where the weights are derived from the responses to a survey sent to experts in major cities. In particular, we collected feedback from the Metro Planning Organizations (MPOs) and Chief Information Officers (CIOs) of 13 major cities. We then predicted the readiness of 52 US cities using various machine learning algorithms and selected decision trees for our final prediction based on its performance. We trained our models using real data collected from the 13 US cities and data from other sources.

The contributions of this research are summarized as follows: 
- We identify three key elements divided into 16 factors necessary to measure a city's readiness towards CAVs.

- We define a numerical CAV readiness index as a weighted average of all 16 factors.

- We determine the weighting factors from the analysis of 13 received responses to a survey sent to planning and technology experts such as MPO officials and CIOs of 52 cities.

- We leverage decisions trees as a machine learning model to predict the overall readiness, as well as the policy, physical and cyber infrastructure readiness of all 52 US cities.

The organization of the paper is as follows. Section 2 presents the factors contributing to CAV readiness and the methodology for computing the proposed readiness index. Section 3 presents our prediction method and results regarding the readiness of 52 US cities. Finally, Section 4 concludes the paper.

\section{CAV READINESS INDEX}

Readiness Indices have been measured before in different domains, such as in health sciences for organ donation [3], technology adoption [4], governance [5, 6], hospitality [7], education [8] and smart urbanization [9]. A recent survey published an autonomous vehicle readiness ranking different countries [10]. Different from their work, we predict the readiness of cities instead of countries towards Connected and Autonomous Vehicles (CAVs). Their work concentrates on the policies and regulations defined by a country's government that can facilitate CAVs in the country. Similarly, INRIX [11] performed a study to rank top US cities for Autonomous Vehicles based on citizens' travel and mobility patterns. They identified issues in current policies and looked into the societal impacts of adopting Autonomous Vehicles. In contrast to the INRIX study, our work identified factors related to not only a city's policies but also a city's physical and cyber infrastructure as these are essential for the safe and efficient operations of CAVs.

We collected real data from the representatives of different cities in the United States regarding the importance of each factor contributing to CAV readiness and then leverage the machine learning algorithm, decision trees to predict the readiness of each city with respect to the CAV deployment.

We sent questionnaires to CIOs and MPO officials from each city asking them to respond on a scale of $1-5$ regarding their opinion on the impact of each element, where a response of 5 corresponds to high impact and 1 corresponds to low impact from the city administration officials. For each element, we computed the average score from the responses of all participants, normalized between $0-1$ to find its respective weight, while the sum of weights in each category sums to unity.

Figure 1 shows the three major categories composing our readiness index, with weights shown in parentheses. We computed these weights by taking the average of the weights suggested individually by each of the experts who completed our questionnaire. Using this procedure, we obtained a weight of 0.31 for policies and regulations, 0.33 for physical infrastructure and 0.36 for cyber infrastructure. Each of these categories is composed by a number of elements, as Figure 1 shows. Each element of a category receives a weight that was calculated using a similar procedure. All elements are summarized in Table 1 with the scoring method described in the last column where each will be explained in the rest of this section.

We believe it would be interesting to emphasize the individual importance of each element irrespective of their relative weight, therefore, in the remainder of this section, we will also mention the individual importance score provided by experts for each element.

In our survey, we requested CIOs and MPO officials from each city to respond on a scale of $1-5$ regarding their opinion on the importance of each element, where a response of 5 corresponds to strong agreement (important) and 1 corresponds to strong disagreement (not important) from the city officials. In the remainder of this section, we will mention the individual score provided by experts for each factor.

In this work, we assume that the MPO officials and CIOs of the most populous cities in the United States are well qualified to assess the importance of different factors we considered in composing the CAV readiness index. Moreover, they are also able to provide concrete data regarding their cities' policies and infrastructure. Therefore, we sent our survey only to these experts. For our future study, we will consider collecting opinions from other segments of the society, e.g., the general public or technologists, and compare them with those from the MPOs and CIOs.

\subsection{CAV Readiness: Policies and Regulations}

A city's readiness towards CAVs is reflected by the dedication of its administration to put forward reforms and policies that can facilitate CAVs' utilization by its citizen. We asked the question of whether "New policies and legislation are needed by the city administration/policy makers to make today's cities ready for CAVs' where on a scale of 1-5, the average response yielded an importance score of 4.16, This is general agreement in the need to revise current policies and legislation to accommodate CAVs.

2.1.1 CAV department. An important factor required in order to define rules and regulations regarding CAVs is for a city to establish a dedicated CAV department within its transportation department. This allows the administration to regulate and modify policies to accommodate CAVs in an efficient manner. For instance, legalizing CAVs to use certain lanes can be part of the CAV department's responsibility. Similarly, regulating licenses and evaluating a CAV's capability to operate on city roads can be efficiently achieved in the existence of such a dedicated department. New Zealand, UK and UAE have already established such departments in their cities [10]. We asked the "Impact of having dedicated team or staff members within the transport department for CAVs" from city administration officials and the average impact score is 3.75 .

2.1.2 CAV investments. The city administration's investment in $\mathrm{CAV}$ related infrastructure is a vital factor to decide the extent the city is ready to facilitate the use of CAVs. The technological constraints today do not allow CAVs to fully operate on current roads and therefore can be driven only in specialized infrastructure with assistance. This requires dedicated testing zones for such vehicles where full external support can be provided for their operation. A simple measure would be to count the amount of money the city administration invested for CAV testing and related pilots/projects. 


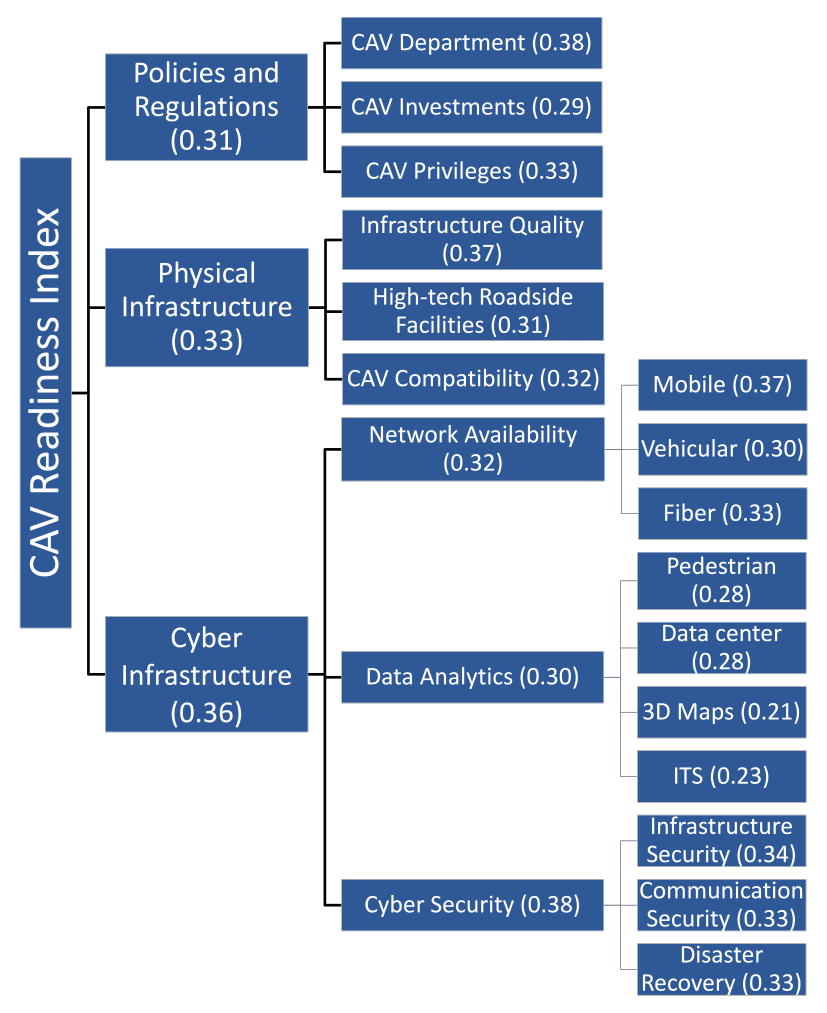

Figure 1: Composition of CAV Readiness Index with each factor's importance weighted by experts shown in parenthesis

As an example of such investment, the Dutch government recently invested 90 million euros to enable more than 1, 000 traffic lights across the country to communicate with vehicles [10].

We asked from the officials from different cities, "How much investments by the city administration in CAV related projects and research impacts its readiness for CAVs?". We obtained an average importance score of 3 .

2.1.3 CAV privileges. This factor measures the rules and regulations defined by the city that prioritize CAVs' usage. One such rule can be allowing CAVs to use High Occupancy Vehicle (HOV) lanes even in the presence of a single person in the vehicle. Similarly, roads in city centers or downtown areas are usually congested and allowing only CAVs to access these roads motivate citizens towards the use of CAVs. In addition to such rules, the city administration can privilege CAVs' to park in dedicated spaces even in congested areas or providing them with tolling privileges compared to humanoperated vehicles. The city administration can also define rules to prioritize CAVs to use safety harbors on highways while ensuring any misuse of safety harbors is prevented. In response to our question on the "Impact of having traffic rules that prioritize CAVs (i.e. in HOV lanes, parking spaces, etc)", the city administrators responded with an average impact score of 3.33 .

\subsection{CAV Readiness: Physical Infrastructure}

The current road infrastructure should be prepared and adapted to the CAV specific needs and therefore, physical infrastructure readiness readiness is an important criterion to consider towards a city's readiness. We asked different city administration officials whether "Improved physical infrastructure (road quality, signage, parking tolling facilitates etc) makes the cities ready for CAVs. The average importance score of 4.1 clearly indicates the perceived importance of a city's physical infrastructure. This section below specifies key factors contributing to the physical Infrastructure readiness.

2.2.1 Infrastructure quality. One of the key factors is the quality of the city's physical infrastructure. Unlike human drivers, the computing and processing capabilities on-board a CAV would require more clear details of the city's physical infrastructure to accurately detect road markings and signage for its driving decisions. Therefore, the city should regularly maintain high-quality road markings and signage by ensuring they do not wear off easily and are clearly detectable. We asked the "Impact of Infrastructure quality (roads, bridges, clear road marking and signage etc) on CAVs" to which a score of 4 suggests a need of maintaining a high quality road infrastructure in order to accommodate CAVs.

One way of quantifying this measure is to evaluate how frequent the city administration inspects the quality of its roads, bridges, road markings, signage and how often it schedules repairs and repainting. Moreover, the number of smart cones and beacons over the total road mileage in the city can also be considered towards the physical infrastructure quality. We consider the existence of 


\begin{tabular}{|c|c|c|c|c|}
\hline Factor & Description & Criteria & Rationale & Scoring \\
\hline \multirow{3}{*}{ Policy } & CAV department & Whether a dedicated CAV department exists & Responsible for CAV rules and regulations & Binary value $\{0,1\}$ for department availability \\
\hline & CAV investments & Investment normalized w.r.t population & Facilitate CAV technological development & Binary value $\{0,1\}$ for available investment \\
\hline & CAV privileges & Amount of rules to prioritize $\mathrm{CAV}$ traffic exist & Incentivize citizens towards $\mathrm{CAV}$ usage & Binary value $\{0,1\}$ if $\mathrm{CAV}$ privileges available \\
\hline \multirow{3}{*}{ Physical Infrastructure } & Infrastructure quality & Infrastructure quality normalized w.r.t road network & CAVs can easily detect road markings/signage & Binary value $\{0,1\}$ for quality inspection availability \\
\hline & High-tech roadside facilities & EV charging stations density w.r.t population & Accommodate large number of Electric CAVs & Value in the interval $[0,1]$ for $\mathrm{EV}$ stations ratio \\
\hline & CAV compatibility & Ratio of CAV compatible infrastructure w.r.t population & CAV compatible facilities require low height/space & Value in the interval $[0,1]$ for facilities w.r.t population \\
\hline \multirow{3}{*}{ Cyber-Network availability } & Mobile Network & City-wide High-speed mobile network coverage & High-speed wireless Internet connectivity for CAVs & Binary value $\{0,1\}$ for for mobile Internet availability \\
\hline & Vehicular Network & City-wide short-range wireless RSUs density & Facilitate short-range V2X and I2V communication & Binary value $\{0,1\}$ for RSU availability \\
\hline & Fiber Network & City-wide optical fiber available w.r.t road network & Facilitate RSUs high-speed Internet connectivity & Binary value $\{0,1\}$ for fiber network availability \\
\hline \multirow{4}{*}{ Cyber-Data analytics } & Pedestrian & Whether pedestrian detection technology available & Assist CAV promptly detect pedestrians & Binary value $\{0,1\}$ for technology availability \\
\hline & Data center & Whether a data center is available & Accommodate local control center for CAVs & Binary value $\{0,1\}$ for data center availability \\
\hline & 3D Maps & Whether 3D maps are available & Assist CAVs for driving & Binary value $\{0,1\}$ for maps availability \\
\hline & ITS & Whether an ITS system is available & Assist CAVs for driving & Binary value $\{0,1\}$ for ITS availability \\
\hline \multirow{3}{*}{ Cyber security } & Infrastructure security & Whether ITS infrastructure secure & Physical and cyber attacks prevention & Binary value $\{0,1\}$ for ITS security system availability \\
\hline & Communication security & Measures to secure communication exist & Jamming and hacking prevention & Binary value $\{0,1\}$ for secure communication measure availability \\
\hline & Disaster recovery & Whether a disaster response/recovery system exists & Responsible for recovery after disaster/attack & Binary value $\{0,1\}$ for disaster recovery system availability \\
\hline
\end{tabular}

Table 1: CAV Readiness Index Summary

regular inspections scheduled by the transportation department as a binary variable contributing to this factor.

2.2.2 High-tech roadside facilities. A large number of high-tech roadside facilities such as EV charging stations would be required to accomodate CAVs as we expect most CAVs to also be electric vehicles (EVs). Thus, a city is ready for CAVs if it has an abundance of EV charging stations. Abundance is not simply the number of charging stations. A city might have a large number of EV stations compared to other cities but the number might not be sufficient for its population. Therefore, this factor should be normalized with respect to the city's population. The publicly available data on the number of EV charging stations normalized with respect to population for each city is collected from [12] . We asked experts, "How much having large number of electric vehicles charging stations in the city impact its readiness for CAVs", in response to which an impact score of 3.52 was provided.

2.2.3 CAV compatibility. The city should design new CAV compatible parking facilities and road lanes. Additionally, the existence of dedicated CAV-only pick-up and drop-off areas contribute to the infrastructure readiness as CAVs operate differently compared to human drivers and having dedicated areas will ease their usage. It is also important to find out whether the city has developed any CAVonly pick-up/drop-off areas in crowded areas. In our questionnaire, we inquired regarding the "Impact of having new CAV compatible lanes, parking and tolling facilities in the city" to which an average impact score of 3.57 was provided by city administration officials.

\subsection{CAV Readiness: Cyber Infrastructure}

The technological evolution of today's cyber infrastructure plays a key role in facilitating CAVs' usage. In this section, we identified three key cyber infrastructure elements that can be considered towards a city's CAV readiness index. In response to our comment "Improved cyber infrastructure (Mobile network coverage, ITS infrastructure, etc ) makes the cities ready for CAVs", the city administration provided an average importance score of 4.3 , thus indicating that cities should prioritize making improvements to their cyber infrastructure to help facilitate CAVs on their roads.

2.3.1 Network availability. An important aspect of the cyber infrastructure readiness is seamless Internet availability in order to efficiently move CAV related data. To this extent, we consider the following three types of network availability in the city.
- Mobile data network availability: CAVs can use the high speed 4G/LTE or the upcoming 5G technology links provided by the mobile network providers to communicate with the infrastructure network. High-speed Internet connectivity allows them to efficiently send or receive safety critical information to a centralized control center on the go. Therefore, a city-wide mobile network coverage greatly contributes to the CAVs' readiness index. One way of finding this measure is to find Internet coverage or the availability of city-wide Wifi network coverage with respect to different population clusters. In our survey, we inquired regarding the availability of Mobile Internet coverage in the city. The "Impact of having a mobile network" was scored 4.7 by expert policy planners.

- Vehicular communication technology availability: This measure considers the different V2V (Vehicle to Vehicle) and V2I (Vehicle to Infrastructure) communication technology developed till date and the deployment of any such technology by the city administration. For instance, one measure is to find the amount of deployed Road Side Units (RSUs) available in the city with respect to the population. RSUs are connected to the infrastructure network using short or long-range wireless communication technology and can help collect and diffuse data to and from CAVs on the roads.Additionally, a city administration can consider the total number of registered vehicles equipped with longrange (4G/LTE) and short-range V2I communication devices enabling local V2V content exchange. We collected data regarding RSUs deployment in each of the considered cities. An average impact score of 3.65 was obtained regarding the "Impact of having Road Side Units (RSUs) for short range communication between vehicles and infrastructure".

- Fiber network availability: The availability of a city-wide optical fiber network is also important for CAVs' connectivity. A city can connect all the RSUs using such a network where the information from the CAVs can be efficiently transmitted through these RSUs. The length of fiber cable per population can be considered to measure this element, however, due to the unavailability of such data, we considered the availability of fiber Internet in each of the considered cities as a binary variable. The "Impact of having a city-wide optical fiber network" was scored 4.05 . 
2.3.2 Data analytics. We live in the era of Big-data and the constantly generated and consumed data on urban streets plays a vital role in facilitating CAVs. We asked different city administration officials and they agree with us by providing an importance score of 4.2 on a scale of $1-5$ that "Efficient Intelligent Transportation System (ITS) measures should be available" for data analytics in the urban environment.

- Pedestrian/obstacle detection technology for infrastructure: Roadside cameras alone are a great source of urban Big-data and can be leveraged to assist CAVs' operation. Therefore, the amount of roadside cameras or other infrastructure equipped with state-of-the-art pedestrian detection technology is an important factor that facilitates CAVs' driving and maneuvering. This requires intelligent software and machine learning algorithms at the infrastructure that can process data from cameras, extract useful information, and communicate it to the CAVs in real time. A large number of cameras equipped with such intelligent pedestrian detection techniques can further enhance pedestrian detection capabilities by using $\mathrm{I} 2 \mathrm{~V}$ communication thereby informing CAVs regarding any hazard in a timely manner. In our survey, we collected the pedestrian detection technology availability information as a binary variable and asked regarding the "Impact of pedestrian detection technology availability in the Intelligent Transportation System (ITS) infrastructure" and an average impact score of 4.15 was provided.

- Local data center availability: Beside roadside cameras, CAVs will also generate and consume lots of data. To efficiently process and analyze such large amount of Big data, there should be a local data center within the city. Such a data center can also be served as a control center for all ITS related operations and will assist CAVs' while driving. We collected data on a data center availability as a binary variable in each of the considered cities and asked regarding, "The impact of data center availability within the city to store, process and analyze the data collected from urban streets". An average impact score of 4.1 was provided by experts.

- 3D maps of the road network: One aspect towards CAVs' readiness is the availability of high resolution and 3D maps of the city. CAVs can store such maps locally with no Internet requirements in bad weather where there is low Internet connectivity. We collected the 3D maps availability information as a binary variable for each city and asked, "How much having city-wide 3D maps are important for its readiness towards CAVs?", An average impact score of 3.4 was provided.

- ITS availability in the city: There already exists a satisfactory ITS system in some cities. However, the availability of such resources should be enhanced such as the installation of sensor-equipped cones and beacons at work sites along with having roadside information communication devices where needed to be able to interact with CAVs. We collected the availability of sensor-equipped cones and beacons as a binary variable for each city and asked experts: "How much availability of sensor-equipped cones, beacons and roadside information communication devices impacts a city's readiness" and in response, an impact score of 3.6 was provided.

2.3.3 Cyber security. One important aspect of CAVs' operation is securing the ITS infrastructure and communication against either physical or cyber attacks. The city should also be able to handle a disaster situation as a result of hacking or jamming its cyber infrastructure in order to ensure the safety of its citizens. In response to "Measures are needed for cities to cope with CAV related cyber security issues (cater threats such as hacking/jamming of the CAV communication, etc)", an impact score of 4.2 was provided.

- Infrastructure security: The security of a city's ITS infrastructure against any possible cyber attacks (i.e. hacking of the traffic lights, etc) is a factor in CAV readiness.We collected data on the availability of such measures as a binary variable and inquired regarding the "Importance of securing the available ITS infrastructure (cameras, RSU etc) from possible physical and cyber attacks". An impact score of 4.9 was provided by city administration officials.

- Communication security: CAVs' greatly rely on communications with other nearby vehicles and RSUs and jamming or hacking V2X or I2V communication can be deadly. Therefore, we consider a binary variable as a measure for securing the short-range communication between CAVs and infrastructure towards the readiness index of the city. The "Importance of securing the short range communication between CAVs and infrastructure against hacking and jamming" had an impact score of 4.85 , thus requires high attention from policy makers.

- Disaster recovery: The city administration is usually prepared for natural disasters. However, disasters and security threats caused by CAVs have not been discovered yet. The readiness for CAVs should consider how well the city is prepared when anything goes wrong and its capability to respond to potential CAV related disaster and security threat. Thus, we consider the existence of such a measure as a binary variable that contributes towards a city's CAV readiness. For the question on the "Importance of availability of disaster response unit for recovery after disaster/attack related to CAVs", the average impact score of 4.9 was provided.

\section{CAV READY CITIES}

We predicted the readiness of 52 US cities based on the collected data regarding the proposed 16 factors. The Chief Information Officers (CIOs) and (Metropolitan Planning Organization (MPO) officials of the 52 cities were contacted and 13 provided the information required to compute the readiness index. The readiness of the remaining cities is predicted using Decision tree model. We employ the leave-one-out as our cross-validation method where, for validation, we use the readiness score computed based on the responses from the officials of each of the compared cities.

\subsection{Predicting Readiness}

We use Decision trees to predict the policy, physical and cyber infrastructure readiness of the 52 cities. The features we used to correlate similar cities are (i) population count in 2017 and the (ii) 


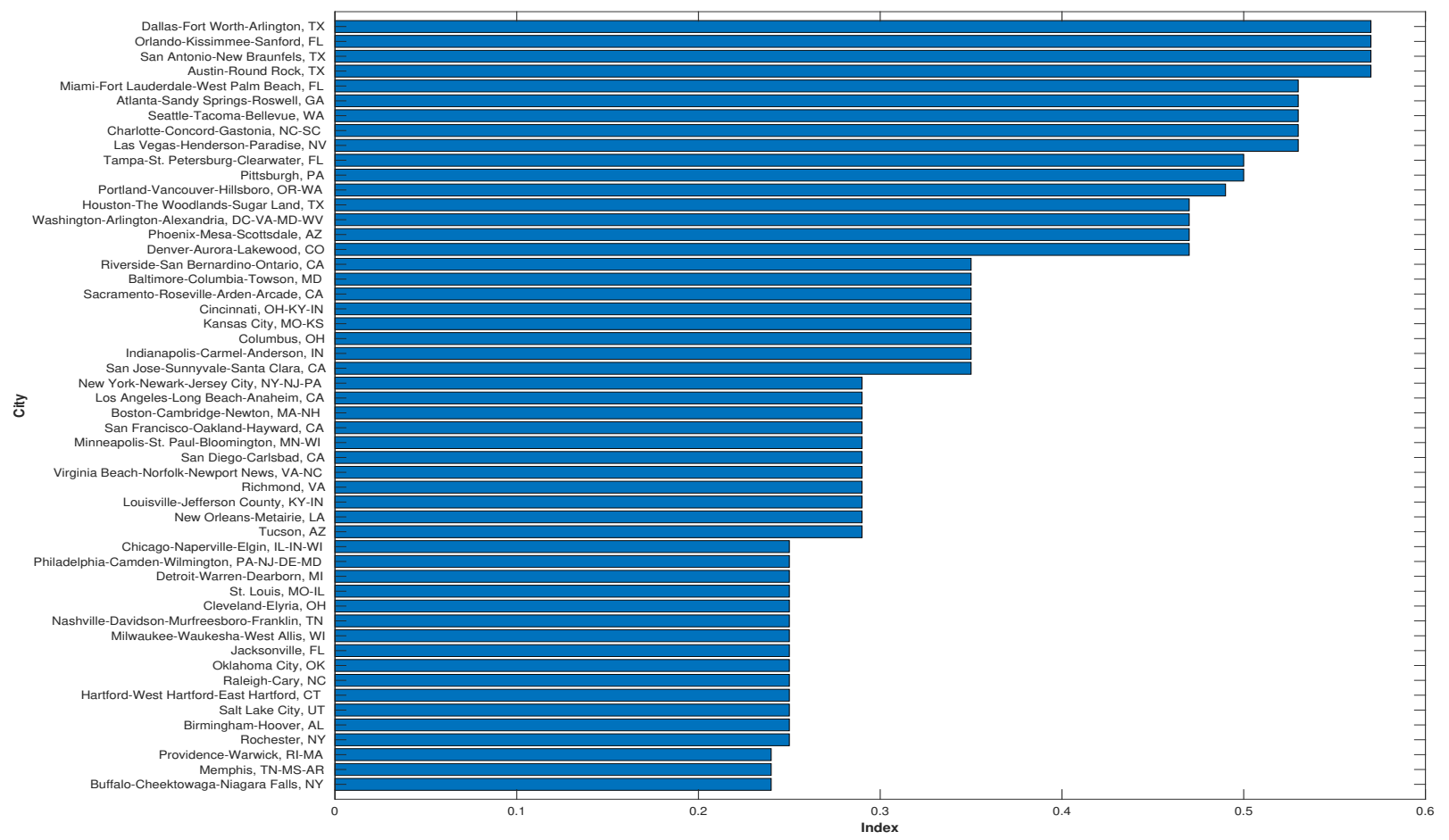

Figure 2: Overall readiness

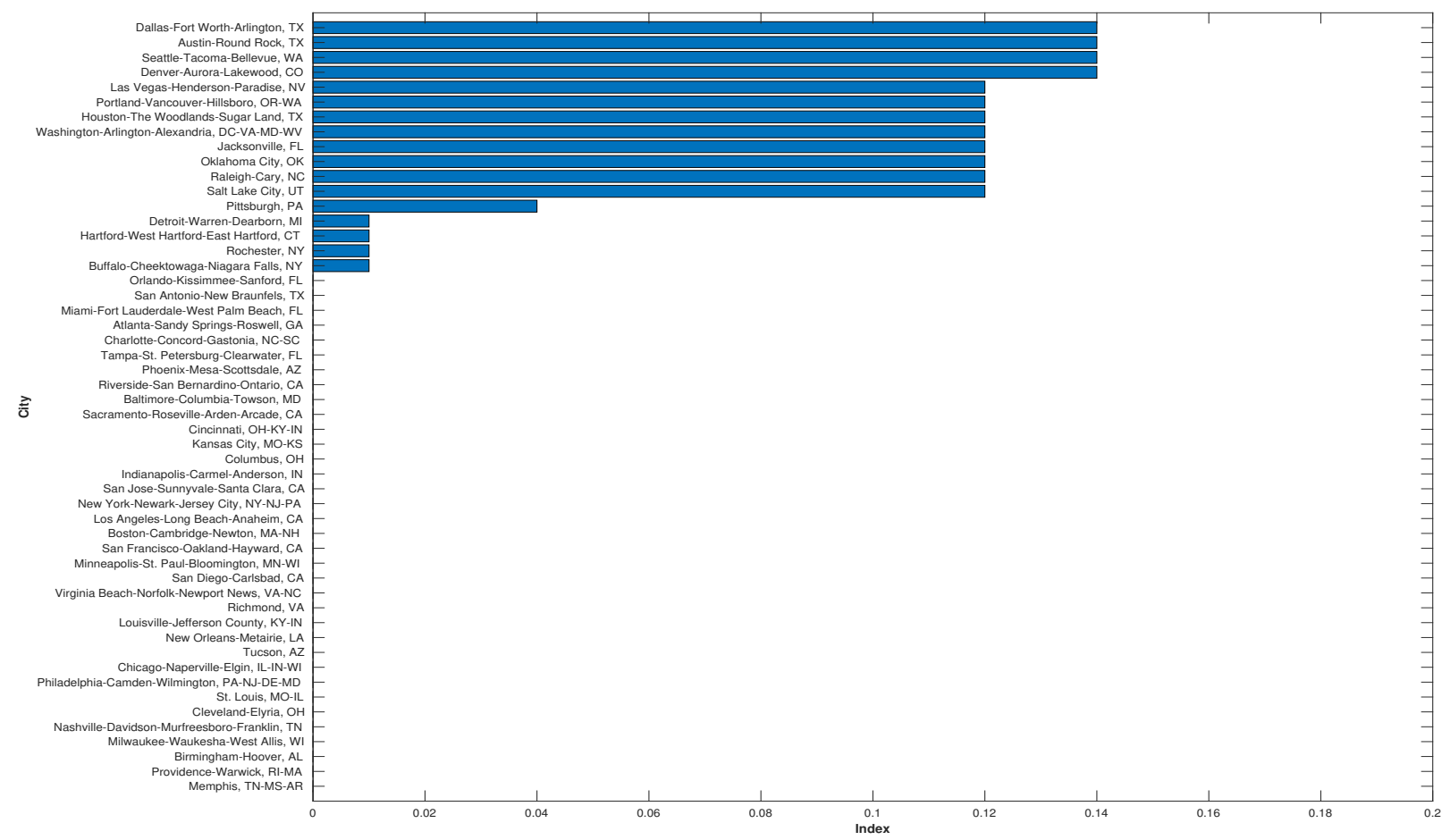

Figure 3: Policy 


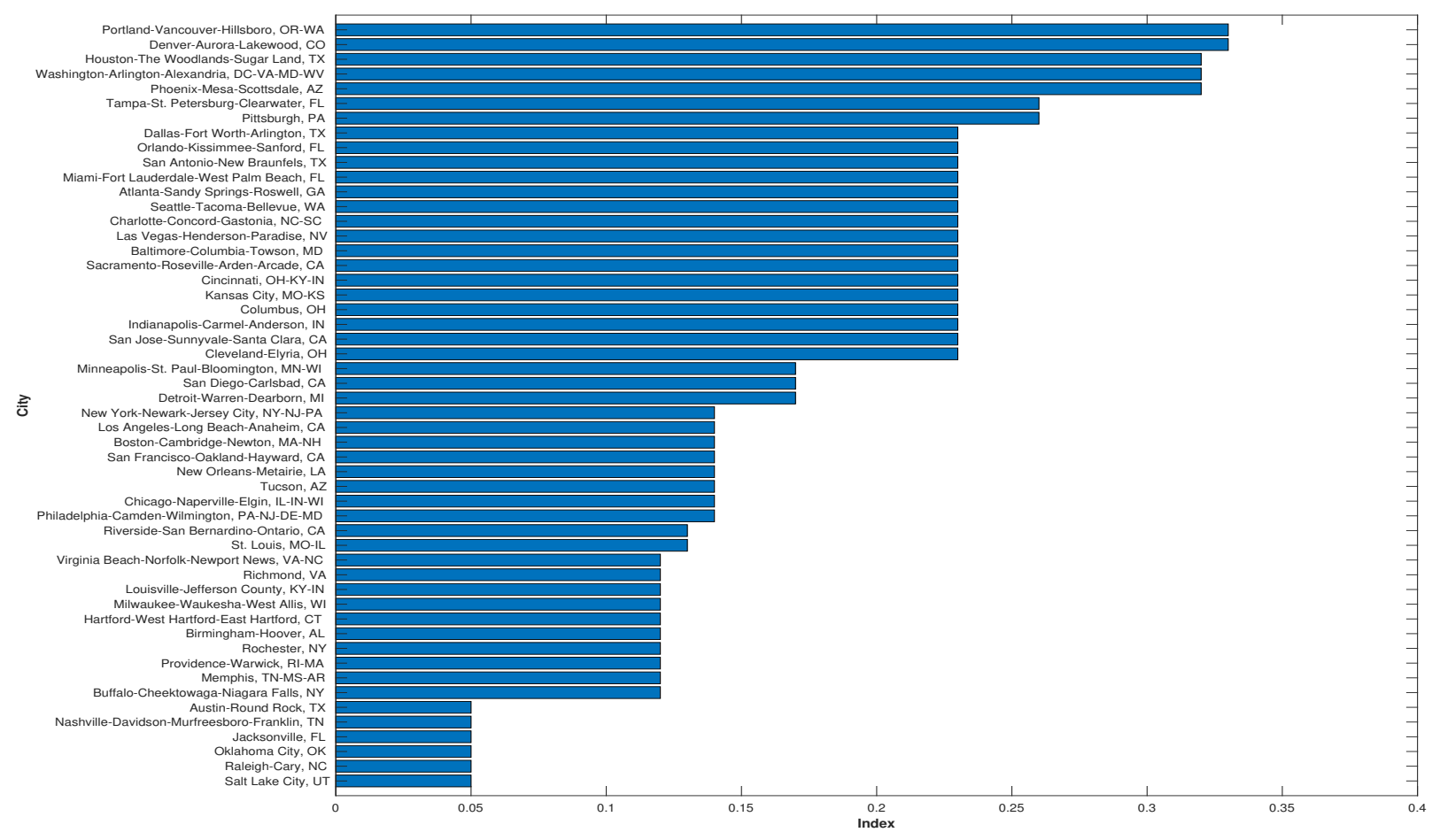

Figure 4: Physical infrastructure

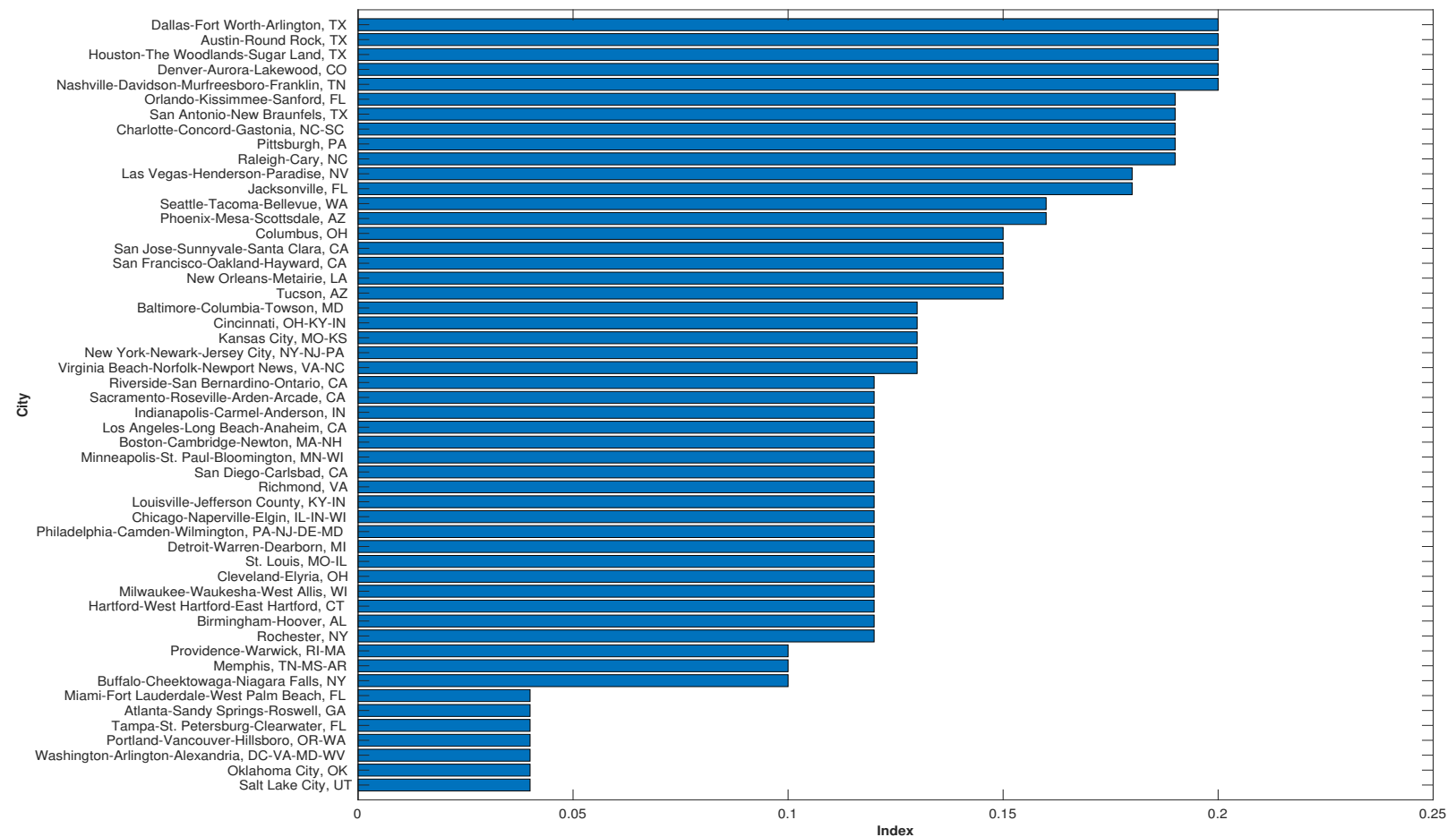

Figure 5: Cyber infrastructure 
growth in population between 2010 and 2017. Further, we consider (iii) the city's Gross Domestic Product (GDP) in 2017 and its (iv) GDP growth between 2012 and 2017 along its (v) GDP per capita in 2017 as a feature to classify similar cities in order to predict their readiness.

We cross-validated the predicted values from the decision tree model by comparing it with the readiness computed from the responses collected for the 13 cities This makes 25\% of training data with ground truth information for the decision tree to predict the readiness values for the remaining $75 \%$ cities as testing data with leave one out method for cross-validation.

Figure 2 shows the predicted CAV readiness index for the 52 cities. Overall, we observe that the Dallas, Texas region is most ready for CAVs. Further, Figures 3, 4 and 5 show the cities readiness with respect to policy, physical, and cyber infrastructure. Different cities lead in each category. Dallas also has the highest policy readiness together with Austin, Seattle and Denver. However, given the policy readiness score of around 0.14 for most of the cities, no city can be declared ready with respect to their policies and regulations measures. Thus, there is a need to devise new policies in order to facilitate CAVs. Physical infrastructure results in Figure 4 suggest that Portland, Oregon is more ready as there are already deploying infrastructure for CAV testing [13]. Similarly we see Phoenex, Arizona among the top five cities with its physical infrastructure ready since this is the city where Waymo is testing its self-driving taxi service [14].

Finally, Figure 5 shows the cyber infrastructure readiness score for the 52 cities. Dallas, Texas slightly leads with a relatively similar score as other cities.

Overall, we observe that none of the cities exceeds a cyber infrastructure readiness score of 0.2 , suggesting the need for measures to be taken by cities in improving their cyber infrastructure to support CAVs.

\section{CONCLUSIONS}

The advent of CAVs in future smart cities is likely. There is a lack of study on whether cities are ready for connected and autonomous vehicles or are at the discretion of car manufacturers and technology companies. Therefore, in this paper, we proposed a new readiness index as a measure of current cities readiness towards CAVs. A numerical index is derived from the weighted average of 16 factors with respect to three key elements, a city's policies, its physical and cyber infrastructure. We leveraged decision tree as the machine learning model to predict the readiness index for 52 cities based on the real training data collected from 13 cities in the United States.

Results from the comparison of different cities readiness index show that each city's readiness with respect to different aspects differs with most cities far from ready, though, large cities, for instance, Dallas, Texas with its high resources seems relatively more ready. However, there is still a need for cities to consider improving their policies/regulations, physical infrastructure and in particular their cyber infrastructure in order to facilitate CAVs on their roads.

\section{REFERENCES}

[1] Ahmadreza Talebian and Sabyasachee Mishra. Predicting the adoption of connected autonomous vehicles: A new approach based on the theory of diffusion of innovations. Transportation Research Part C: Emerging Technologies, 95:363-380, 2018.

[2] Jesse Simpson, Sabyasachee Mishra, Ahmadreza Talebian, and Mihalis M Golias. Disaggregated prediction of adoption rate of autonomous trucks by freight organizations. Technical report, 2019.

[3] Christopher Rice and Judith Tamburlin. A confirmatory analysis of the organ donation readiness index: measuring the potential for organ donations among african americans. Research on Social Work Practice, 14(4):295-303, 2004.

[4] Ananthanarayanan Parasuraman and Charles L Colby. An updated and streamlined technology readiness index: Tri 2.0. Fournal of service research, 18(1):59-74, 2015.

[5] Anteneh Ayanso, Dipanjan Chatterjee, and Danny I Cho. E-government readiness index: A methodology and analysis. Government Information Quarterly, 28(4): 522-532, 2011.

[6] UN Department of Economic and Social Affairs. Global e-government readiness report: Toward access for opportunity, 2004. URL https://unpan1.un.org/intradoc/ groups/public/documents/un/unpan019207.pdf.

[7] Liana Victorino, Ekaterina Karniouchina, and Rohit Verma. Exploring the use of the abbreviated technology readiness index for hotel customer segmentation. Cornell Hospitality Quarterly, 50(3):342-359, 2009.

[8] M Semih Summak, Murat Bağlıbel, and Mustafa Samancıoğlu. Technology readiness of primary school teachers: A case study in turkey. Procedia-Social and Behavioral Sciences, 2(2):2671-2675, 2010.

[9] Mohamed Anwer Zayed. Towards an index of city readiness for cycling. International journal of transportation science and technology, 5(3):210-225, 2016.

[10] KPMG. Autonomous vehicles readiness index, 2019. URL https: //home.kpmg/content/dam/kpmg/nl/pdf/2019/sector/autonomous-vehiclesreadiness-index-2019.pdf.

[11] INRIX. Highly autonomous vehicle city evaluation, 2017. URL http://inrix.com/wp-content/uploads/2017/03/INRIX-Automated-VehicleStudy-2017_FINAL.pdf.

[12] ChargeHub. Ev charging stations map, 2019. URL https://chargehub.com/en/ charging-stations-map.html.

[13] Portland. Cav testing site, 2019. URL https://www.pressherald.com/2018/07/17/ portland-selected-for-autonomous-driving-test-site/.

[14] Waymo. Self-driving taxi testing location, 2019. URL https://waymo.com/tech/. 